\title{
AN ACCESSORY STUDY OF "PHONETIC SYMBOLISM"
}

\author{
By Madison Bentley and Edith J. Varon, Cornell University
}

In 1929 Sapir published a brief study from which he concluded that certain vocalic and consonantal sounds have a definite symbolic significance unrelated to the associative and linguistic value commonly attached to words. ${ }^{1}$ His method consisted in presenting two 'unfamiliar' words (e.g. mal and mil), to which a meaning (e.g. 'table') was arbitrarily attached. His subjects reported whether mal symbolized a larger or a smaller table when compared with the word mil. The results indicated that there was a tendency for vowels near the a-end of the vocalic scale to imply 'largeness' and for those near the $i$-end to imply 'smallness,' quite apart from linguistic uses of these sounds. A like reference was also discovered for certain consonants.

In the immediately preceding article in this Journal S. S. Newman has, at the instance of Professor Sapir, again attacked the problem of phonetic symbolism, applying to his research a method of statistical treatment not used in the earlier study, and using a much larger body of material than the earlier study commanded. ${ }^{2}$ Newman's results generally confirm Sapir's earlier conclusions, making the interpretation much more specific. Newman again makes phonetic symbolism apply differentially to 'large' and 'small' and adds also the contrasting pair 'bright' and 'dark.' The distribution of his vowel-sounds and consonant-sounds upon the scale of magnitude (large-small) and the scale of brilliance (dark-light) leads him to believe that it is determined by such mechanical factors as position of the tongue in articulation, resonance within the mouth, the size of the oral cavity, and length of the vowel sound. Some differences in these factors with respect to their influence upon the two scales are thought to be significant.

Upon the appearance of the first article, we were led to undertake a bit of experimentation upon the subject, partly to adapt the method to the psychological laboratory and partly to discover how far "phonetic

* Accepted for publication October 1, 1931.

$\rightarrow$ E. Sapir, A study in phonetic symbolism, J. Exper. Psychol., 12, 1929, 225-239.

${ }^{2}$ S.' S. Newman, Further experiments in phonetic symbolism, this JourNaL, 45 , 1933, 53-75. 
symbolism" was actually inherent in the sounds used and how far it arbitrarily laid hold of variety in the make-up of the non-linguistic 'words' and turned them perforce into degrees of 'magnitude.' We used a small number of students trained in research, and we devised various means for extracting size or magnitude, without hinting at it, from the presented sounds. Furthermore, we wished to discover-in the event of a more or less,' 'a this or that'-whether such an intimation of degree or of contrasting quality pertained specifically to spatial determinations. In certain series, therefore, we added to size the nine categories of angularity, foolishness, endurance, liquidity, sentimental attachment, motion, noisiness, solidity, and strength.

Method I. (One category word: 'large.') Forty series of 10 nonsense syllables each (vowel between two variable consonants) were made up and presented to each of three $O$ s $(D, G$, and $L)$.

The $O$ s were graduate students trained in the procedures of the experimental laboratory. They knew therefore how to observe under prescribed instructions and how to make relevant and precise reports. Each series contained either two or three familiar words and seven or eight non-linguistic (n-l) sounds. Of the latter, two were critical; i.e. they contained vowels taken from near the ends of Sapir's vocalic scales. One vowel was a (Ger. Mann), the other $i$ (Fr. fini). The completing consonants were varied. The critical sounds always appeared between 'nonsense' sounds. In the first 20 series they were separated from each other; in the last 20 . series they directly followed each other, as a possible enhancement of the "contrasting' vowels. $O$ was screened from $E$, that mouth-setting, posture, or gesture might not be effective.

$O$ was instructed to respond promptly with the first sound that came after hearing a given sound pronounced by $E .^{3}$ Since rhymes and assonances were at first frequently given in response, $O$ was presently instructed either to give a synonym or otherwise to express the meaning of the heard sound, but to avoid rhymes and similar-sounding words. The forty series were three times given (in changed order) to each $O(120$ series $\times 10$ sounds each $\times 3$ Os $=3600$ sounds altogether $)$. Sometimes we found the sound completed to form a word (saf-Sappho); sometimes an onomatopoeic meaning came (zin-noise of a flying missile; vub-a frog

\footnotetext{
${ }^{3}$ Although one experimenter (Varon) uttered all the sounds used in this study, the precise and consistent character of the non-linguistic sounds proved to be difficult. As Dr. Sapir has urged in correspondence, the common use by him and us of identical phonetic symbols offers no guarantee that the same sounds were actually employed. We suggested master-records on phonographic disks; but the matter could not be arranged. There remains the possibility, then, that certain differences between his results (together with Newman's) and ours may be due in part to unlikenesses in the actual sounds uttered. Of course the Sapir experiments have the advantage of expert knowledge of phonetic quality and quantity. At the same time, we must see that, however unfaithful our sounds may have been to the high phonetic standards set, we have without doubt attained such differences in the "mechanical factors" assumed by Newman (supra p. 61) to be operative as should make our results as well as his significant for the problem of "phonetic symbolism."
} 
sound; zuv-a soft, gentle something), and sometimes a near-lying word came (kir -care).

No responses definitely indicated, however, that such a factor as phonetic symbolization was present, at least in a degree that led to a responsesound that suggested size or any determination of magnitude. Of course this negative outcome does not imply that magnitudes and differences of magnitude might not be forced from the sounds under other conditions. 'Feeling significances' (Sapir) might be potentially at hand but too weak to compete with 'reproductive tendencies' (whatever these theoretical conditions of vocalization may be!) under our conditions of presentation. The results do suggest, however, that such sounds as we have employed do not spontaneously lead to other sounds (under the common conditions of 'free association') which denote or hint at spatial magnitude or its qualifications.

Method II. (10 category-words, each followed by an n-l sound.) Here we extended our search to the 10 categories named above. The method approached one step nearer that of Sapir (first article), but still preserved notable differences.

The general procedure was to sound one of the 10 category-words (angular, foolish, large, lasting, liquid, loving, moving, noisy, solid or strong) and then to sound a non-linguistic (n-1) sound with the instruction to report whether this sound implied some degree of the first or of its opposite (e.g. large or small). If it did, the report was to be 'slightly,' 'moderately,' or 'very;' if it did not, the report was to be 'unrelated.' Thus the second sound in each pair was non-linguistic $(n-1)$. Choice was made here from a list of 50 monosyllables. For constructing these the vowel $e$ (Eng. met) was added to the $a$ and $i$ used under Method I. Sometimes the initial consonantal sound was kept constant (e.g. gam, gem, gim), thus providing an opportunity to compare results with a single context for unlike vowels. Each series of ten pairs (e.g. for a pair, angular-kig) contained 4 a-sounds, 4 $i$-sounds, and $2 e$-sounds. The category-words and the n-l sounds were combined in a haphazard order. As each category-word was given with each n-1 sound, 500 combinations were presented to each $O$ (10 words $\times 50$ following-n-1-sounds $\times 3$ $O s=1500$ instances in all). The $O$ s were $D, G$, and $L$ as before.

Of the 1500 reports under this method 450 indicated some kind of relation (called positive report) between the category word and the n-1 sound following it in each pair.

Agreements and disagreements among the positive reports stand as follows for the 450 instances;

$\begin{array}{crrccc} & & & \text { Disagreement } & \text { Agreement } \\ \text { Reported by } 10 & 2 \text { Os } & 3 \text { Os } & \text { (in direction) } & \text { Total } \\ 202 & 174 & 18 & 56 & 450\end{array}$


Thus well above two-fifths (202) of the cases of positive relation were reports made by a single $O$. Two $O s$ agreed upon approximately fourninths (192) of the cases; all three Os agreed only 18 times. Disagreement as to amount or direction of the relation was shown in 56 cases. These disagreements are illustrated by lel, reported as 'moderately large' $(D)$, and as 'moderately small' $(G)$; wim reported as 'slightly weak' $(G)$, and as 'equally weak and strong' $(D)$.

A little less than one-third of the whole number of reports (450:1500) indicate, then, that, to the $n-1$ sounds used, the quality expressed in the category-words was applied by one or more of our Os, under the conditions imposed by our experiments. Upon most (960) of the 1050 'unrelated' or negative reports, two of the three $O s$ agree. This high agreement upon the want of relation may be as significant as the positive reports. It seems to imply that a good many of the total n-l sounds used (i.e. the total 'nonsense syllable') symbolically represent no qualification of the categories used.

As regards our positive reports, an analysis of the results shows that a large number of our $50 \mathrm{n}-1$ sounds led to some degree of relationship where each one of our 10 categories was used. With respect to the degree of relationship (slightly, moderate, very), few (only 8 altogether) 'very' reports were made. Nearly all the reports alleged a 'slight' relationship. Of all 10 categories, the fewest positive reports were given with the meaning solid. Some notable differences with respect to the vowel of the n-l sound were observable. Thus the positive 'angular' reports were chiefly returned with $a$ - and $e$-sounds, seldom with an $i$-sound. The 'solid' reports seldom came with an $e$-sound or the 'strong' reports with $a$ and $e$. The total number of positive reports is distributed as follows among the 10 categories; angular 35 , foolish 48 , large 32 , lasting 42 , liquid 41 , loving 41 , moving 34 , noisy 32 , solid 23 , and strong 35 . It is possible, therefore, to report a relation (of more or less) over a wide range of diverse qualities.

Method III. (5 categories and their opposites, with n-l sounds in pairs.) As a still closer approach to the original method of Sapir, we next followed our category-word with two n-1 sounds. The $O$ was instructed to report which (if either) of the two n-l sounds more closely approximated the meaning of the category-word just given.

Five categories were used; foolish, angular, liquid, large, and strong. First, one of these category-words was pronounced (e.g. 'foolish'), then (at a suitable interval) two n-l sounds (e.g. 'zat,' 'fim') in quick succession and with an even inflection and intonation. The sounds $o$ (go) and $u$ (tufa) were added in this series. We considered that, although our results would be few and not admissible 
to statistical treatment, they would be obtained with trained and practiced Os (the 3 Os of Methods I and II), working under evener and more constant conditions than could well be commanded in a group, and that they might give hints of selectiveness in the phonetic scales as Sapir and Newman have observed with fairly large groups of Ss. Twenty combinations with each category-word were made, in both temporal orders for the n-l sounds.

In the 300 pairings ( $5 \times 20 \times 3$ Os), some degree of relationship was reported 165 times and for all 5 categories. In only 7 cases was the degree reported as 'very,' and as 'moderate' only 17 times. All the others are 'slight.' Comments by all Os bear out the suspicion that the relation was usually forced and that, when it was reportable, the difference in 'size,' 'liquidity,' etc., as between 'zat' and 'riv,' 'lel' and 'duf,' etc., was both doubtful and obscure. The case is different with Newman, who asked his. $S$ s whether this or that $\mathrm{n}-1$ sound was the larger or the smaller 'horse,' and so on. In his case the two sounds were arbitrarily given the meaning 'horse' and the larger (or smaller) arbitrary 'horse-sound' was then checked. Our Os were left free to discover that either or neither of the $\mathrm{n}-1$ sounds applied to size, strength, and the other category-words. In both studies it is obvious that the formal and the occasional instructions are weighted-albeit by different devices - to force a meaning into an $n-1$ sound and then to suggest that this meaning admitted of degree. Most of the Sapir-Newman results bear upon the "magnitude-pattern" forced upon names, activities and adjectival qualities. Newman added (Experiment II) a "darkness pattern." Our employment of a larger number of "patterns" (category-words) makes it again obvious that $O$ s may be forced to report differentially upon degree in many other contexts as well. It is strikingly attested also by the fact that our Os reported 21 opposites for 'large,' 'liquid,' and 'strong;' i.e. certain non-linguistics (n-1) were reported as 'small' (6), as 'solid' (7), and as 'weak' (8), although these words had not been prescribed. Of these opposites, 14 were $e$ - and $i$-sounds, and 6 were $a$-, $o$-, and $u$-sounds. But 'liquidity' and 'strength' favored the $i$-sounds quite as much as size did.

Method IV. (Group experiment: 3 categories and their opposites; partially forced meanings.) We now used the 'arbitrary meaning' device of Sapir. Twenty-six members of a class doing drill-experiments in psychology were used. The general setting was experimental, for a part of the laboratory-period was devoted to our observations. In order to enhance the experimental setting, the instruction as given by Sapir was somewhat modified.

Sapir found that his exercise with high school students "seemed to be enjoyed by the great majority of his subjects" (high-school students) "as a rather interesting 
game." Although his conditions and instructions were not quite clear, he seems to have prearranged that two sounds, about to be uttered, were to be given an arbitrary meaning (e.g. mal and mil were to be given the meaning 'table'). Then when these sounds were given, the $S$ checked in a column marked "large" if the first of the sounds "symbolized the larger reference," and in a column marked "small" if the first sound symbolized the smaller reference. Whether 'copying' was controlled among the $500 \mathrm{Ss}$ does not appear. To eliminate errors of time-order, in our experiments, and to make sure that the cumulative results of $O$ 's own checking should not influence each succeeding check-mark, the written result was immediately removed from his view as soon as it was finished. In column-checking a common form of error from occasional instruction is to even the number of entries in both (or all) the columns in the serial run. Whether the children observed Sapir's face when he uttered the sounds, does not appear. The open-mouth continental $a$ might be quite enough to swing the $a$-words to the 'large' column. That is one reason for our invariable use of a screen. Another possible source of degreedifference in $\mathrm{n}-\mathrm{l}$ sounds emanates from the hearer. Sapir dwells upon this possibility, factoring it into auditory and kinaesthetic components. Unfortunately, this source was not sufficiently controlled, either in his and Newman's investigations nor in ours. How much all our Os used their vocalizing apparatus-either for incipient sounds or for 'gesturing' - no one knows. Five minutes' attention to the strains and postures incident to making brief vocal sounds will convince one that there is here plenty of material which, given a linguistic turn, will easily absorb 'degrees,' not only of magnitude but also of many other qualifications. We used $36 \mathrm{n}-1$ paired combinations. First, the four sounds fam, jaf, maf, and vag were paired with fim, iif, miv, and vig. Thus 'contrasting' vowels were systematically distributed and combined. In the second place, we added sounds to pair off all the vowels among themselves (as in Method III). Finally, consonantal variations. were used with each vowel (e.g. bav-pav, bav-pag, pav-pag, etc.) to discover the specific effect of the consonants. Haphazard order was followed throughout. There were 4 series of 9 sound-pairs each. Three categories (angular, large, bard) and their common opposites (round, small, soft) were used with all $36 \mathrm{n}-1$ pairs. The order of utterance in each case was: (1) a pair of $n-1$; (2) object-, action-, or quality-word; (3) category-word. An instance is (1) fam-fim; (2) cabin; (3) which is the larger cabin? The vocal presentation had been preceded by the general instruction: "I shall utter two sounds (e.g. fam-fim), then an arbitrary meaning which you are to apply to them (e.g. cabin), then ask you to compare the two sounds, if you can, with respect to some attribute (e.g. size)." Here we departed from Sapir's procedure in leaving the possibility that the two sounds might not be comparable with respect to the attribute. We wished to force the comparison as little as possible.

Our tables show, as the Chicago studies had shown, a persistent inequality in the 'largeness' of the various vowel-sounds. Here are the results from a paired comparison of four a-sounds and four $i$-sounds with the arbitrary meaning 'table.'

${ }^{4}$ Op. cit., 229. 


\begin{tabular}{cccc}
\multicolumn{4}{c}{ Object $=$ Table (large and small) } \\
A-sounds & I-sounds \\
(fam, jaf, mav, vag) & (fim, gif, miv, vig) & totals \\
larger' & 176 & 46 & 222 \\
smaller' & 41 & 189 & 230 \\
totals & 217 & - & 452
\end{tabular}

The a-sounds are larger than the $i$-sounds (table) in the approximate ratio of 4:1. Reading down each column, the large-small ratio for $a$ sounds (table) is also approximately 4:1. The reverse relation holds also for the $i$-sounds. While calculated reliabilities would have little significance for our small number of observations and of observers, the internal consistency is striking.

It is worth nothing that, in size-of-table, the a-sounds are conspicuously more in number of 'large' reports than the $i$-sounds, and conspicuously less in number of 'small' reports in every one of the sound-pairs compared. With respect to size, then, a (as in Ger. Mann) is consistently larger (and less small) than $i$ (as in Fr. fini).

We may add similar results with the same paired sounds when the arbitrary object is 'table' (angular and round) and is 'chair' (hard and soft).

A-sounds

Object $=$ Table $($ angular and round)

(same as above)

'more angular'

'rounder'

65

199

I-sounds

(same as above) totals

totals

264

193

258

254

55

512

Object $=$ Chair (hard and soft)

A-sounds

(same as above)

'harder'

'softer'

totals
I-sounds

(same as above)

164

88

252 totals

252

250

502

As regards angularity we find again a decided difference as between the two n-1 sounds. For both sounds, the ratios for angularity and round stand $1: 3$ and $3: 1$. Again the totals for the two sounds are about the same (264, 248.) 
With respect to hardness, $a$ is softer (and less hard) than $i$. An unlikeness in clarity of difference-with respect to the three qualities-appears in the three approximate ratios, 4:1 (size), 3:1 (roundness), and 2:1 (softness). That the $e$ used (as in Eng. met) stands, in the respects noted, near our $i$ is suggested by the fact that fam is reported larger than fem (table) and softer (and less hard) than fem (chair) by fairly large ratios (cf. Newman's quantitative results).

It appears at once that the positive reports form only a moderate fraction of the whole number. We have omitted all unrelated reports, as well as reports of same and doubtful.

Although Sapir and Newman are not clear upon the point, they seem to say that their method of forced meaning led only and always to the report of unlike degree. Our method left $O$ free to note or to fail to note the applicability of the category to the comparison in question. The forcing instruction, in such cases, is bound to increase the number of positive reports, but it may well affect validity also. One wonders whether Sapir's Ss may not, in 'playing their game,' have soon learned under forcing to apply a half-formulated rule with respect to sounds and magnitudes. Every one familiar with the conduct of psychophysical methods knows how readily even highly trained $O$ s pick up devices which relieve them of making bona fide comparisons.

Of course the discovery that arbitrary meanings and forced choices may lead invariably to a comparative report of, say, magnitude does not prove that the 'magnitude' dimension came out of the n-1 sound in the form of a 'phonetic symbol.' It only shows that, under the conditions, the Ss apply, with more or less consistency, some sort of difference in the two n-l sounds to the problem proposed by the experimenter. In our own experiments we have found that the qualifications suggested by 'arbitrary meanings' come out in proportion to the amount of forcing. 'Free association' brought only a few conventional responses, either through a linguistic similar (melsmell), a completion (saf-sappho), or an obvious onomatopoeia (bahbaa!). No real 'phonetic symbolism' in Sapir's sense appeared. The positive report did appear when degree in some scale was suggested and prescribed.

Sapir's fascinating problem lies on the border-line between linguistic material and non-symbolic sounds. It is natural that a student of languages and of phonetics should incline the problem toward his own field. The articles constantly refer to 'words' even where non-linguistic sounds ( $\mathrm{n}-\mathrm{l}$, in our own shorthand) were used. It may be instructive, therefore, to drop for the moment this context and to regard the sounds as auditory or other objects of perception. This would be the psychologist's natural inclination. Then it would appear that the simplest and best-ordered series of 
sounds (the tonal series) is a one-dimensional series, proceeding from low, grave, voluminous, dull, and weak members, at the one end, to high, acute, small, bright, and strong members, at the other. After decades of conjecture and experiment upon the possible 'associative' character of certain of these qualifications, it is generally agreed among psychologists that some such set of aspects is actually attributive and therefore inherent in the tones and that they gradually change from one end of the series to the other. As everyone knows, the effectiveness and significance of speech, music, and natural sounds constantly exemplify - and often voluntarily use-these attributive qualifications of the simpler sounds. Intensity is another instructive attribute. It is capable of fine gradation and may directly (i.e. without symbolization) be apprehended as strength. Once more, there is in all these attributive qualifications a principle of surrogative function by way of which any one may stand for any other and may be interchangeably used. Thus a decided intensive difference will readily adopt the context of size or of gravity and so stand for a 'contrast' in volume or in pitch. In fact, it requires long and rigorous training to distinguish modifications in pitch, volume, dullness and intensity. ${ }^{5}$

When we turn to the simpler noises, we find virtually the same set of attributes; though there is probably no actual identity, quality for quality, in the two series. If then we proceed to those complexer sounds which form the basis of linguistic communication, we find again the same sort of distinguishing characteristics. But here the case is considerably complicated by hundreds of diverse fusions of individual sounds. Nevertheless, it is a commonplace to the students of language and of music that the simpler vocal sounds partake of the general nature and constitution of their fused and temporally colligated members (compare tone and note, note and vowel, vowel and larger phonetic unit).

As it happens, Sapir lighted upon a qualification for 'phonetic symbolism' which is exceedingly prominent in the simple tonal system; namely, voluminousness (magnitude), and Newman has added a second, namely, brightness (dull and bright). Possibly no other choice would have turned out to be so significant. The positions in the tonal scale from which the main ingredients of the vocalic sounds are drawn well illustrate obvious spacings with regard both to 'magnitude' and to 'brightness.'

Now these vocalic characteristics, and others like them in consonantal elements, pertain to the sounds as sounds. They attain a 'phonetic' or 'symbolic' signification only when we transfer them to other contexts. It

${ }^{\circ}$ Cf. R. H. Gundlach and M. Bentley, The dependence of tonal attributes upon phase, this JOURNAL, 42, 1930, 519.543. 
appears that they have not generally been useful on the meaning side of language, albeit they possess high aesthetic value both in speech and in music.

It does appear from these studies that certain tonal, vocalic, and consonantal characteristics, which likewise bear a fixed relation to physical sources of sound and also to the human productive mechanisms (position of tongue, resonance, size of oral cavity, etc.), as Newman and Sapir have pointed out, are so well graduated as to be usable when forced by artifice into the representation of size and of various other concepts and categories as well. Nevertheless, there seems to be insufficient evidence that these graded attributes of sound carry in their own right (so to say) a symbolic reference. Newman discovered, ${ }^{6}$ as we might expect, that linguistic usage derives its verbal and syntactical meanings (true symbolization) from other sources. The case is probably the same for colors, where also we have heard a good deal of 'symbol' and 'fixed emotive significance.' Even gustatory and tactual qualities are useful indicators of many things, but chiefly by an adventitious overlay of reference upon qualitative specificity.

As a demonstration of the use of attributive characteristics of simple vocal and instrumental sounds in discriminations of the sort exemplified by Sapir and Newman, we presented to an introductory class in psychology (327 university students) 5 pairs of n-l sounds, three of them vocal (fam-jif, vig-vam and miv-vag) and two instrumental, the first a succession of simple tones from tuning forks ( 512 vs. and 2304 vs.) and the other pair two fairly simple noises of the explosive sort (the first produced by the thud of a padded mallet on heavy wood, the other the crack of a small pith-hammer upon a wooden surface).

The following instruction preceded the giving of the sound-pairs. "I shall utter two sounds in succession from behind this screen, first warning you with a 'ready.' As you hear the two sounds, consider which of them appears (just as a sound) larger (lighter, softer, smaller) than the other." The first vocal pair was compared for largeness, the second for lightness (as contrasted with darkness), and the third for softness. The two tuning-fork tones were compared for largeness and the two noises for smallness. The results follow.

\begin{tabular}{|c|c|c|c|c|}
\hline Reported & First & Second & Neither & Totals \\
\hline fam-jif ('larger'). & 165 & 83 & 79 & 327 \\
\hline vig-vam ('lighter') & 240 & 62 & 25 & 327 \\
\hline miv-vag ('softer') & 184 & 72 & 71 & 327 \\
\hline tones ('larger') & 265 & 38 & 24 & 327 \\
\hline \multirow[t]{2}{*}{ noises ('smaller') } & 15 & 304 & 8 & 327 \\
\hline & 869 & 559 & 207 & 1635 \\
\hline
\end{tabular}

${ }^{6}$ Supra pp. 71-75. 
It appears, therefore, that untrained observers find it possible to distinguish these characteristics in $\mathrm{n}-\mathrm{l}$ vocal and instrumental sounds without any such linguistic reference as Sapir and Newman used. We have no reason to believe that there was involved in these comparisons anything like a 'feeling significance' or a 'symbolic reference.' The comparisons seem to have been based directly upon inherent differences within the sounds themselves. It is notable that the greatest difference between the first and the second sounds (as implied in the distribution of the reports) occurs in the simplest sounds, where the lower tone is reported 'larger' than the higher in a ratio of approximately $7: 1$ and the higher noise is reported 'smaller' than the lower in a ratio of approximately 20:1.

Again, 'magnitude' (and perhaps other graded qualities) may find a direct means of representation in the immediate visual perception of an articulating organism. The open mouth, the stretched jaw, the tense lip, all doubtless carry spatial and dynamic meanings which immediately enter into the specific characterization of the vocalized sound as heard.

Finally, the act of sound-production itself, which includes the feel of the tongue, lips, jaw, chest, and other parts of the body, contributes its large share to the sound as heard. As the context is favorable, this tactual and kinaesthetic component, variable in many directions and through wide ranges, probably serves in quite essential ways to convey its own meaning.

In all these cases, the materials serve, not by any inherent power of specific symbolization but rather as a graded and labile system, to inform the occasion-whether linguistic or not-in some appropriate and significant manner.

Psychology is deeply indebted to these phonetic studies for suggesting a number of interesting and significant problems which are now ripe for further research. 